\title{
Chronology of the three-body dissociation of ${ }^{8} \mathrm{He}$
}

\author{
B. Laurent田, F.M. Marqués ${ }^{1}$, C. Angulo'他, N.I. Ashwood ${ }^{3}$, \\ M.J.G. Borge ${ }^{4}$, V. Bouchat ${ }^{5}$, W.N. Catford ${ }^{6}$, N.M. Clarke $^{3}$, \\ N. Curtis ${ }^{3}$, M. Freer ${ }^{3}$, F. Hanappe ${ }^{5}$, V. Kinnard ${ }^{5}$,

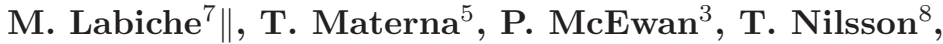 \\ A. Ninane ${ }^{2}$, G. Normand 4 , N.A. Orr ${ }^{1}$, S.D. Pain ${ }^{6}$, \\ E. Prokhorova ${ }^{5}$, L. Stuttgé ${ }^{9}$, C. Timis ${ }^{6}$ \\ ${ }^{1}$ LPC Caen, Normandie Université, ENSICAEN, Université de Caen, \\ CNRS/IN2P3, Caen, France \\ ${ }^{2} \mathrm{CRC} / \mathrm{UCL}$, Louvain-la-Neuve, Belgium \\ ${ }^{3}$ School of Physics and Astronomy, University of Birmingham, Birmingham \\ B15 2TT, UK \\ ${ }^{4}$ Instituto de Estructura de la Materia, CSIC, E-28006 Madrid, Spain \\ ${ }^{5}$ Université Libre de Bruxelles, CP 226, B-1050 Bruxelles, Belgium \\ ${ }^{6}$ Department of Physics, University of Surrey, Guildford, Surrey, GU2 7XH, UK \\ ${ }^{7}$ Department of Engineering and Science, University of the West of Scotland, \\ Paisley PA1 2BE, UK \\ ${ }^{8}$ ISOLDE, CERN, CH-1211 Geneva 23, Switzerland \\ ${ }^{9}$ Institut de Recherche Subatomique, IN2P3-CNRS, Université Louis Pasteur, \\ BP 28, F-67037 Strasbourg cedex, France \\ E-mail: marques@lpccaen.in2p3.fr \\ 13 February 2019
}

\begin{abstract}
The space and time configurations of the dissociation of ${ }^{8} \mathrm{He}$ into ${ }^{6} \mathrm{He}+n+n$, on $\mathrm{C}$ and $\mathrm{Pb}$ targets, have been explored simultaneously for the first time. The final-state interactions in the $n-n$ and ${ }^{6} \mathrm{He}-n$ channels are successfully described within a model that considers independent emission of neutrons from a Gaussian volume with a given lifetime. The dissociation on $\mathrm{C}$ target exhibits a dominant sequential decay through the ground state of ${ }^{7} \mathrm{He}$, consistent with neutrons being emitted from a Gaussian volume of $r_{n n}^{\mathrm{rms}}=7.3 \pm 0.6 \mathrm{fm}$ with a $n$ - $n$ delay in the sequential channel of $1400 \pm 400 \mathrm{fm} / c$, in agreement with the lifetime of ${ }^{7} \mathrm{He}$. The lower-statistics data on $\mathrm{Pb}$ target correspond mainly to direct breakup, and are well described using the $n-n$ volume measured, without any $n-n$ delay. The validity of the phenomenological model used is discussed.
\end{abstract}

Keywords: neutron correlations, three-body decays, halo nuclei

$\ddagger$ Present address: CEA, DAM, DIF, F-91297 Arpajon, France.

$\S$ Present address: SCK-CEN, Boeretang 200, 2400 Mol, Belgium.

|| Present address: STFC Daresbury Laboratory, Daresbury, Warrington WA4 4AD, UK.

I Present address: GANIL, Caen, France. 


\section{Introduction}

Along the neutron dripline some nuclear systems develop an extreme clustering structure already in their ground state, in which the weakly bound neutrons form a halo around the rest of the system, or core 11. The most exciting of these are the Borromean two-neutron halo systems, ${ }^{6} \mathrm{He},{ }^{11} \mathrm{Li}$ and ${ }^{14} \mathrm{Be}$, exhibiting a bound core- $n$ - $n$ structure where all the two-body subsystems are unbound 2. As such, these systems are unique for the study of three-body correlations. Other heavier candidates, ${ }^{17,19} \mathrm{~B}$ and ${ }^{22} \mathrm{C}$, have not been explored in detail yet, and the structure of ${ }^{8} \mathrm{He}$ seems to be dominated by an $\alpha$ core plus four neutrons [3].

The breakup of these genuine three-body systems unveils a complex interplay between the nature of their decay, either direct or sequential [4, 5, and the resonances and final-state interactions (FSI) in the two-body channels. Compared to systems involving charged particles, two-neutron haloes convey a clearer picture of the decay due to the absence of Coulomb forces. The population of core- $n$ resonances dictates the sequential nature of the decay, and the $n$ - $n$ interaction modifies their final state at low relative momentum [6, 7, 8, In particular, Ref. 8] links this modification with the space-time proximity of the neutrons, under the hypothesis of independent emission from a Gaussian source, by exploiting the principle that the effect of the short-range nuclear force will be stronger at shorter distances.

The dissociation in the field of a heavy target can be used at intermediate energies to induce direct breakup of two-neutron haloes [9, 10]. In that case, no core- $n$ resonances are populated and, according to Ref. [8, the only parameter characterizing the decay should be the space dimension of the neutron emission. This approach was used to estimate the average $n$ $n$ separation at breakup in ${ }^{6} \mathrm{He},{ }^{11} \mathrm{Li}$ and ${ }^{14} \mathrm{Be}$, leading to $r_{n n}^{\mathrm{rms}}$ values of $6-7 \mathrm{fm}$ in agreement with predictions from three-body models [11. However, in the case of ${ }^{14} \mathrm{Be}$ it was found that breakup on a lighter target introduced a sequential channel through various resonances in ${ }^{13} \mathrm{Be}$ [12, that induced an average delay between the emission of the neutrons and thus reduced the $n-n$ signal. The delay, $150_{-150}^{+250} \mathrm{fm} / c$, was attributed to a series of resonances in the ${ }^{13} \mathrm{Be}$ system, their number and energy being not completely resolved.

A similar decrease of the FSI effects in a nucleon pair due to sequential decay was recently observed in two very different scenarios, the two-neutron decay from the continuum of ${ }^{18} \mathrm{C}$ and ${ }^{20} \mathrm{O}[13]$ and the twoproton decay of unbound states of ${ }^{22} \mathrm{Mg}$ and ${ }^{23} \mathrm{Al}$ [14. In both works, the direct and sequential channels were clearly identified, and the analysis using the simple FSI model with a space-time Gaussian source lead to source sizes of the order of a nucleus of mass 20. The delays obtained, however, could not be assigned to individual intermediate resonances and, as in Ref. [12, were only considered qualitatively.

The possibility to measure a neutron delay generated by the well-defined lifetime of an intermediate core- $n$ resonance represents a unique opportunity to map the space-time decay of a three-body system. Ideally, the core- $n$ subsystem should have one state and if possible with narrow width (long lifetime), that would induce a significant delay between the emission of the neutrons. While ${ }^{8} \mathrm{He}$ is understood as a four-neutron halo or skin [3, its three-body ${ }^{6} \mathrm{He}+n+n$ breakup channel exhibits the characteristics noted above: only one state is known in ${ }^{7} \mathrm{He}$ decaying into ${ }^{6} \mathrm{He}+n$, and it is narrow enough $(\Gamma=150 \pm 20 \mathrm{keV}$ [15]) to significantly delay the emission of the second neutron $(\hbar / \Gamma=1320 \pm 180 \mathrm{fm} / c)$.

In this Letter, we report on the dissociation of a ${ }^{8} \mathrm{He}$ beam into ${ }^{6} \mathrm{He}+n+n$ on $\mathrm{C}$ and $\mathrm{Pb}$ targets. The formation of ${ }^{7} \mathrm{He}$ has been clearly identified in Dalitz plots, showing that the breakup on $\mathrm{C}$ target is mainly sequential and goes through the ground state of ${ }^{7} \mathrm{He}$. The description of the three-body final state with the FSI model used in Refs. [12, 13] leads for the first time to the simultaneous measurement of the $n-n$ volume at breakup and the delay between the emission of the neutrons, the latter being in very good agreement with the lifetime of ${ }^{7} \mathrm{He}$. These observations are confirmed in the breakup on $\mathrm{Pb}$ target, in which no ${ }^{7} \mathrm{He}$ is observed and that is successfully described by the direct emission of the neutrons from the same volume and without any delay.

\section{Experiment}

A ${ }^{8} \mathrm{He}$ secondary beam of $15 \mathrm{MeV} /$ nucleon and $10^{4}$ pps was produced at GANIL using SPIRAL, and was tracked onto the breakup targets $\left(95 \mathrm{mg} / \mathrm{cm}^{2} \mathrm{C}\right.$ and $284 \mathrm{mg} / \mathrm{cm}^{2} \mathrm{~Pb}$ ) on a particle-by-particle basis using a thin plastic detector $(100 \mu \mathrm{m}$ of $\mathrm{BC} 408)$ and a drift chamber at $11 \mathrm{~cm}$ upstream of the target. The ${ }^{6} \mathrm{He}$ fragments were identified using two $500 \mu \mathrm{m}$ Si strip detectors and sixteen $2.5 \mathrm{~cm}$ CsI crystals from the CHARISSA collaboration inside the reaction chamber, and the neutrons detected using 90 elements of the DEMON array, placed in a staggered arrangement at forward angles in order to minimize the contribution of cross-talk (the scattering of one neutron through several detectors) [16].

With the momenta of the core $+n+n$ breakup fragments, we calculate the two and three-body invariant masses $M_{c n}$ and $M_{c n n}$, and then the decay energy of the system $E_{d}=M_{c n n}-m_{c}-2 m_{n}$ and the relative core- $n$ energy $E_{c n}=M_{c n}-m_{c}-m_{n}$ (Fig. (1). Although $E_{d}$ peaks at about $2 \mathrm{MeV}$, the energy of a 

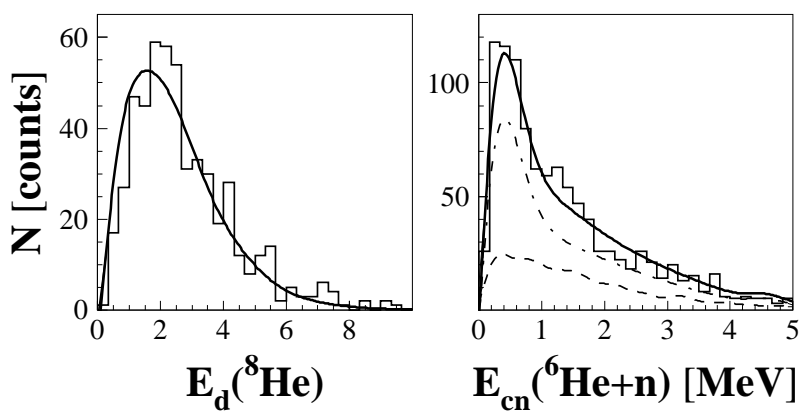

Figure 1. Reconstructed decay energy of the ${ }^{6} \mathrm{He}+n+n$ system (left) and the ${ }^{6} \mathrm{He}+n$ subsystem (right), for the breakup of ${ }^{8} \mathrm{He}$ on a $\mathrm{C}$ target. The solid lines correspond to the input (left) and result (right) of the simulation described in the text, decomposed on the right panel into its sequential decay (dot-dashed) and direct breakup (dashed) components.

resonance observed in a previous work [17, we have not assumed the population of individual resonances due to the limited acceptance (that decreases beyond $2 \mathrm{MeV}$ ) and resolution (about $1.5 \mathrm{MeV}$ FWHM at $2 \mathrm{MeV}$ ), and have focused on the final-state correlations emerging from this continuum energy distribution.

The interacting phase-space model used was introduced in Ref. [12. In brief, the experimental $E_{d}$ distribution is used as input to generate events following three-body or twice two-body phase space [18, respectively for direct or sequential decay. The latter is described as the breakup into one neutron and a core- $n$ resonance, with a relative energy $E_{c n}$ given by a Breit-Wigner line-shape with energy-dependent width of parameters $(E, \Gamma)$, which is then allowed to decay into the core plus the second neutron. The $n$ - $n$ FSI is introduced via a probability to accept the event as a function of the $n$ - $n$ relative momentum $q_{n n}=$ $\left|\vec{p}_{n_{1}}-\vec{p}_{n_{2}}\right|$ following the form of the $n-n$ correlation function $C_{n n}$ [8, 11, defined by the neutron emission volume. The final momenta are filtered through a simulation, including all experimental effects [11, 16, and the different observables are reconstructed.

\section{The decay model}

In the analog case of the decay of core- $p$ - $p$ systems, the correlations in the $p-p$ channel have been often interpreted in terms of the microscopic structure of the two-proton emitter, like for example in the $2 p$ decay of ${ }^{6} \mathrm{Be}$ and ${ }^{45} \mathrm{Fe}[19$. Within this formalism, the relative $p-p$ energy is given by the configuration mixing of the three-body wave function of the emitter, that may lead to an 'anti-correlation' (strength at high relative energy, back-to-back emission) or even to oscillatory patterns [19, 20]. Recently, a similar formalism has been proposed for core- $n-n$ decays 21 .

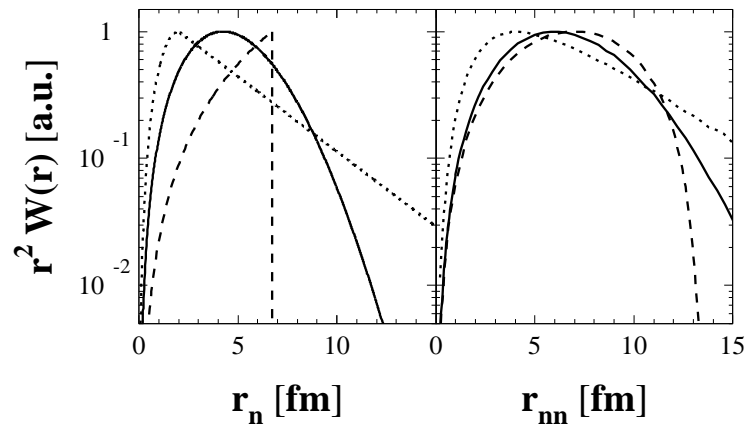

Figure 2. Density distributions (left) corresponding to a Gaussian of sigma $3 \mathrm{fm}$ (solid), a sphere (dashed), and a Yukawa (dotted line), all with the same rms radius. The distributions of relative distance between two independent particles within these volumes are shown on the right.

However, the core- $n$ - $n$ final states measured to date do not exhibit any such patterns. In the $2 n$ decay from the continuum of ${ }^{5} \mathrm{H}\left[22,23,{ }^{6} \mathrm{He}[11,22\right.$, ${ }^{8} \mathrm{He}$ (this work), ${ }^{11} \mathrm{Li}$ [11, 24, ${ }^{14} \mathrm{Be}$ 11, 12, ${ }^{16} \mathrm{Be}$ 25], ${ }^{18} \mathrm{C}$ and ${ }^{20} \mathrm{O}$ [13, the strength is systematically located at low $n-n$ energy, as predicted by Refs. 6, 7]. This low-energy signal is originated by the $s$-wave $n$ $n$ interaction, and is in fact used in the reaction with the simplest two-neutron final state, ${ }^{2} \mathrm{H}\left(\pi^{-}, \gamma\right) 2 n$, to determine the $n$ - $n$ scattering length $a_{n n}$ [26, 27]. In that system, the geometry of the process is well known (given by the deuteron wave function) and the only free parameter in the description of the low $q_{n n}$ signal (or its counterpart at high photon energy) is $a_{n n}$.

Ref. [8] extends the formalism of Refs. [6, 7] by taking into account the influence of the two-nucleon proximity on the effects of their interaction (see appendix for the relevant formulas). It considers the effect of the $s$-wave scattering amplitude, dominant at low energies, on a pair of nucleons separated by a fourmomentum distance, and integrates it over a source in space and time. The resulting factor becomes a probability distribution that modulates phase space, as in Ref. 27]. This model described accurately the lowenergy peaks observed in the $n$ - $n$ final state of previous works [1, 12, 13, 22, 24, 25, although contrary to Refs. 26, 27, the value of $a_{n n}$ was fixed and it was the size of the source that was varied.

The formalism of Ref. 8 was, however, developed for a Gaussian source emitting independent neutrons. Obviously, we do not pretend that the wave function of ${ }^{8} \mathrm{He}$ is Gaussian, nor the two valence neutrons independent. First, the fact that the neutrons move independently in a Gaussian source was in part exploited in Ref. 8] for analytical ease, as in that case the distribution of relative distance is also Gaussian. However, $C_{n n}$ does not depend on each neutron's position in the source but on their relative distance, so 
we can directly choose the shape of the latter as input of the model, without any hypothesis on the overall matter distribution of ${ }^{8} \mathrm{He}$. Moreover, the distributions of relative distance $W\left(r_{n n}\right)$ in general soften the shape of the individual distributions $W\left(r_{n}\right)$, as can be seen in the examples of Fig. 2 for three very different shapes. This may explain in part why the Gaussian hypothesis has been able to describe reasonably well the final states of previous works, even for systems that were not supposed to be Gaussian.

A second approximation of Ref. [8] is the neglect of internal momentum correlations of the form $W\left(r_{n n}, q_{n n}\right)$. We will assume that those potential correlations are small, or that they have a negligible impact on the correlation factor after averaging over the whole source. The ability of the model to describe, at least at first order, the very specific channel subject of this work represents a severe test that will confirm or refute the validity of these two approximations.

\section{Sequential decay and time}

In Ref. [11, the analysis of the dissociation of halo nuclei on a $\mathrm{Pb}$ target assumed simultaneous emission of both neutrons in the Coulomb field of the target. When there is no emission delay, the correlation function of a Gaussian source becomes analytical A9. If the dissociation is sequential, however, the emission of the neutrons cannot be considered simultaneous and a space-time analysis is needed. The effect of the $n-n$ FSI depends then on the two space and time parameters $\left(r_{0}, \tau_{0}\right)$, that correspond to the sigma values of the Gaussian space-time source (A7/A8 .

In this work, the ${ }^{8} \mathrm{He}$ projectile is considered to be excited by the $\mathrm{C} / \mathrm{Pb}$ target into the continuum, and the unbound ${ }^{6} \mathrm{He}+n+n$ system decays in flight, either directly into the three particles, or through an intermediate ${ }^{6} \mathrm{He}+n$ resonance. Therefore, the only free parameters of our interacting phase-space model are three: $r_{n n}^{\mathrm{rms}}$, the root-mean-square $n$ - $n$ distance $\left(\sqrt{6} r_{0}\right) ; P\left({ }^{7} \mathrm{He}\right)$, the probability of sequential decay through ${ }^{7} \mathrm{He}$ ground state; and $\tau$, the neutron delay introduced by this ${ }^{7} \mathrm{He}$ resonance $\left(\sqrt{2} \tau_{0}\right)$.

The Dalitz plot of the decay after dissociation on the $\mathrm{C}$ target is shown in Fig. 3. as a function of the $n-n$ and ${ }^{6} \mathrm{He}-n$ invariant masses normalized between 0-1 to the available energy $E_{d}[12$. In the absence of interactions/correlations, the whole plot should be populated uniformly, and the projections should follow the phase-space dotted lines. On the other hand, the attractive $n$ - $n$ interaction would overpopulate the low $m_{n n}^{2}$ part, and resonances due to the ${ }^{6} \mathrm{He}-n$ interaction would lead to horizontal bands 12 .

The Dalitz plot exhibits a clear crescent shape as a result of both interactions, a slight increase at low

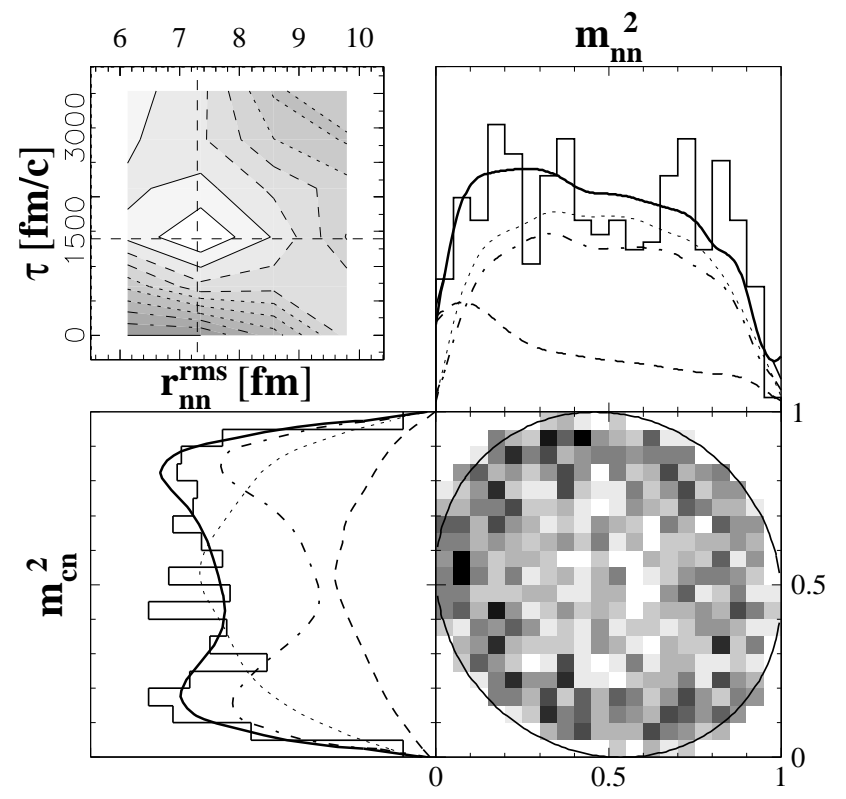

Figure 3. Dalitz plots (core- $n$ versus $n-n$ ), and the projections onto both axes, for the data from the dissociation of ${ }^{8} \mathrm{He}$ into ${ }^{6} \mathrm{He}+n+n$ on a $\mathrm{C}$ target. The lines correspond to the best fit of the simulation (solid), with the contributions of sequential (dot-dashed) and direct (dashed) breakup. The dotted line corresponds to phase space. The inset shows the $\chi^{2}$ between simulations and data as a function of the delay between the neutrons and their average distance at breakup (each contour corresponds to one unit of $\chi^{2}$ ).

$m_{n n}^{2}$ and two horizontal bands at $m_{c n}^{2} \sim 0.15$ and 0.85 , leading to a depletion at the center. This is more easily observed in the projections shown on the same figure, where the slight increase towards $m_{n n}^{2}=0$ and the two peaks on the wings of the $m_{c n}^{2}$ distribution become more evident with respect to the expected phase-space distribution (dotted line). Both interactions are clearly present since none of them (dot-dashed or dashed lines) is able to reproduce the data on its own.

The probability of sequential decay can be easily extracted from the already mentioned characteristic signals of ${ }^{7} \mathrm{He}$ ground state in the ${ }^{6} \mathrm{He}+n$ distributions, the narrow peak in $E_{c n}$ (Fig. 1) and the symmetric wings in $m_{c n}^{2}$ (Fig. 3). A fit of the latter gave a value of $P\left({ }^{7} \mathrm{He}\right)=70_{-5}^{+10} \%$ for the data on $\mathrm{C}$ target. Using this result, we have combined the direct and sequential scenarios and varied the two parameters $\left(r_{n n}^{\mathrm{rms}}, \tau\right)$ describing the space-time characteristics of the neutron source. The determination of the values that describe best the $n-n$ signal in $m_{n n}^{2}$ has been undertaken through the calculation of the $\chi^{2}$ between each simulation and the experimental distribution, leading to the $\chi^{2}$ surface shown on the inset of Fig. 3 . A clear minimum appears at $r_{n n}^{\mathrm{rms}}=7.3 \pm 0.6 \mathrm{fm}$ and $\tau=1400 \pm 400 \mathrm{fm} / c$. The simulation corresponding 


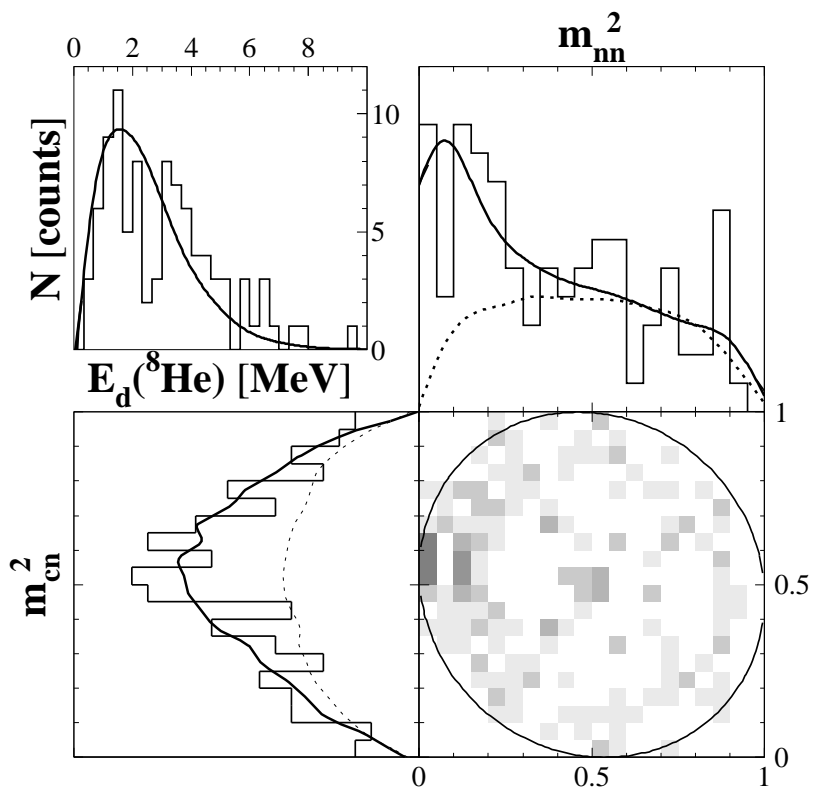

Figure 4. The same Dalitz plot shown in Fig. 3 but for the dissociation of ${ }^{8} \mathrm{He}$ into ${ }^{6} \mathrm{He}+n+n$ on a $\mathrm{Pb}$ target. The solid line corresponds to the simulation of direct breakup with $r_{n n}^{\mathrm{rms}}=7.3 \mathrm{fm}$, and the dotted line to phase space. The inset shows the reconstructed decay energy of the ${ }^{6} \mathrm{He}+n+n$ system, with the solid line being the input of the simulation.

to this minimum is represented by the solid line in the projections of Fig. 3. Note that the $n$ - $n$ FSI acts on all events, depending on the space dimension for direct breakup $\left(r_{n n}^{\mathrm{rms}}\right)$, and on both the space and time dimensions for sequential breakup $\left(r_{n n}^{\mathrm{rms}}, \tau\right)$.

The measured delay between the emission of the neutrons corresponds well to the expected scenario, the lifetime of ${ }^{7} \mathrm{He}(1320 \pm 180 \mathrm{fm} / \mathrm{c})$. Regarding the size of the $n$ - $n$ volume, it should be considered as an average value for the ${ }^{8} \mathrm{He}$ continuum up to $5 \mathrm{MeV}$ beyond the ${ }^{6} \mathrm{He}+n+n$ threshold. We note that this analysis is similar to the one used in Refs. [14, 28, in which the $\chi^{2}$ between the experimental and theoretical correlation functions was minimized in order to extract the size and lifetime of a $p-p$ emitter [14 and of a compound nucleus evaporating neutrons [28].

\section{Direct decay and space}

The dissociation on a $\mathrm{Pb}$ target lead to lower statistics, that prevented the Dalitz plot analysis or the construction of the $\chi^{2}$ surface. Nevertheless, in Fig. 4 we show the plot and its invariant-mass projections in order to compare them to the ones obtained on $\mathrm{C}$ target. In fact, the two peaks observed in the $m_{c n}^{2}$ distribution of Fig. 3 that come from the horizontal bands in the Dalitz plot and are the signature of the sequential part of the decay, disappear completely with
$\mathrm{Pb}$. Instead, we observe the single, wider central peak characteristic of direct breakup, together with a much stronger effect of the $n-n$ FSI at low $m_{n n}^{2}$, clearly above the phase-space distribution (dotted line). We note, however, that the decay energy spectrum (inset) is comparable to the one in Fig. 1, suggesting that we are populating a similar continuum region in ${ }^{8} \mathrm{He}$.

Therefore, if our analysis of the sequential decay on $\mathrm{C}$ target is well founded, and with the $\mathrm{Pb}$ target we have switched off the sequential branch, the data on $\mathrm{Pb}$ should correspond to the direct component present in the $\mathrm{C}$ set. For completeness, we have compared the $\mathrm{Pb}$ data to the simulations of direct breakup using $P\left({ }^{7} \mathrm{He}\right)=0$ and the spatial $n-n$ configuration previously obtained, $r_{n n}^{\mathrm{rms}}=7.3 \mathrm{fm}$ (Fig. 4). The very good agreement with the data suggests that the breakup into ${ }^{6} \mathrm{He}+n+n$ on $\mathrm{Pb}$ does populate states in the continuum of ${ }^{8} \mathrm{He}$ in the same energy range and with similar spatial characteristics, but that decay mainly through the simultaneous emission of both neutrons. This different decay mode can be understood at these bombarding energies by the effect of the stronger Coulomb field of $\mathrm{Pb}$ [9, 10, that by acting only on the core subsystem hinders the probability of core- $n$ resonances (here ${ }^{7} \mathrm{He}$ ) to be formed.

Finally, we have built the experimental $C_{n n}\left(q_{n n}\right)$, the observable used to parametrize the $n$ - $n$ FSI in the model, for the dissociation on C. The experimental distribution was divided by a distribution obtained through event mixing, using the iterative technique described in Ref. [11. The resulting ratio is shown in Fig. 5. The dashed line corresponds to the analytical formula (A9) for $r_{n n}^{\mathrm{rms}}=7.3 \mathrm{fm}$ that rises up to $C_{n n}(0) \approx 11$, a value comparable to the ones measured in Ref. [11. If we use the more general formalism with $\left(r_{n n}^{\mathrm{rms}}, \tau c\right)=(7.3,0) \mathrm{fm}$ for $30 \%$ of events and $(7.3,1400) \mathrm{fm}$ for the other $70 \%$, we obtain the solid line. The agreement with the data is remarkably good, confirming that in this particular case the model does not only reproduce a general trend, but also the fine details of the $n$ - $n$ space-time signal.

\section{Conclusions and outlook}

We have measured the dissociation of ${ }^{8} \mathrm{He}$ into ${ }^{6} \mathrm{He}+n+n$ on $\mathrm{C}$ and $\mathrm{Pb}$ targets. The analysis of triple correlations has given access, for the first time, to the spatial and temporal characteristics of the decay. The use of both targets has proven to be, at these energies, an efficient way to switch on and off a delay between the emission of both neutrons, by selecting a mostly sequential $(\mathrm{C})$ or a mainly direct $(\mathrm{Pb})$ decay mode. The sequential channel is clearly identified through the population of the ground state of ${ }^{7} \mathrm{He}$, and represents a $70 \%$ contribution in the case of the $\mathrm{C}$ target. 


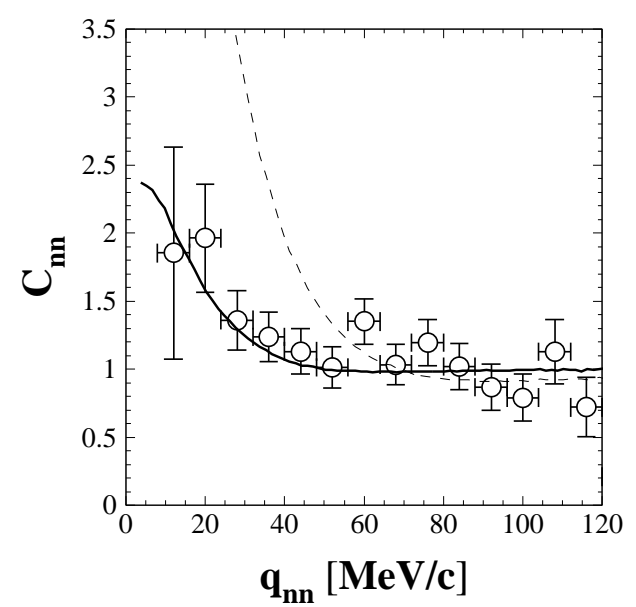

Figure 5. Two-neutron correlation function for the dissociation of ${ }^{8} \mathrm{He}$ into ${ }^{6} \mathrm{He}+n+n$ on a $\mathrm{C}$ target. The dashed line corresponds to direct breakup with $r_{n n}^{\mathrm{rms}}=7.3 \mathrm{fm}$, and the solid line to an additional delay of $\tau=1400 \mathrm{fm} / c$ for $70 \%$ of events.

The parallel analysis of the $n$ - $n$ FSI in both target runs represents a very stringent test of this method as a femtometer and chronometer of $2 n$ decays. In the $\mathrm{C}$ target case, it leads to a sequential decay from a Gaussian volume of $r_{n n}^{\mathrm{rms}}=7.3 \pm 0.6 \mathrm{fm}$, corresponding to states in the continuum of ${ }^{8} \mathrm{He}$ a few $\mathrm{MeV}$ beyond the ${ }^{6} \mathrm{He}+n+n$ threshold, with a delay between the emission of both neutrons of $\tau=1400 \pm 400 \mathrm{fm} / c$, consistent with the lifetime of ${ }^{7} \mathrm{He}$. On the other hand, the results for the $\mathrm{Pb}$ target clearly indicate a different mechanism favoring direct breakup, as expected for the stronger Coulomb field, that is well reproduced using the same $n-n$ volume without any delay.

Regarding the spatial information, it would be interesting to compare this result with theoretical calculations of the $n$ - $n$ distribution in the continuum of ${ }^{8} \mathrm{He}$, similar to those performed for ${ }^{6} \mathrm{He}$ [29]. However, as noted earlier, ${ }^{8} \mathrm{He}$ is better described as a fivebody system. In this respect, it would be interesting to consider a nucleus with a predominant three-body structure, like a heavier two-neutron halo, that at the same time possessed few, narrow states in the core- $n$ subsystem. For example, ${ }^{17} \mathrm{~B}$ exhibits a two-neutron halo 30 and the unbound subsystem ${ }^{16} \mathrm{~B}$ seems to have only one ${ }^{15} \mathrm{~B}-n$ resonance extremely narrow, $\Gamma<$ $100 \mathrm{keV}$ [31, 32. That resonance, if populated through the sequential decay of ${ }^{17} \mathrm{~B}$, would introduce a delay $\tau>2000 \mathrm{fm} / c$ that should strongly hinder any $n-n$ correlation. We note that the breakup of ${ }^{17} \mathrm{~B}$ on $\mathrm{C} / \mathrm{Pb}$ targets has been recently studied at RIKEN [33, and that the analysis of $n$ - $n$ correlations is in progress.

The origin of the difference in the interpretation of these core- $n-n$ final states and the analogous core- $p-p$ ones is not clear yet. While the microscopic structure of the $2 p$ emitter, a rather narrow state, seems to govern the $p$ - $p$ distributions [4, 19, 20], the $n$ - $n$ ones appear to be dominated by the effects of the $s$-wave $n$-n FSI [11, 12, 13, 22, 23, 24, 25, as if the system went directly into the three-body continuum. In fact, in all the neutron works the systems were populated in a broad continuum of decay energies. Even if the $n$ $n$ scattering amplitude is much stronger than the $p-p$ one, unhindered by the Coulomb repulsion that almost cancels the latter, it would be interesting to study these correlations in a core- $n-n$ system with a well-defined energy, that could reveal the eventual breakdown of the model used here and/or the sensitivity to the microscopic structure of the $2 n$-emitter state [21].

\section{Acknowledgments}

The support provided by the technical staff of LPC and the LISE crew is gratefully acknowledged, as are the efforts of the GANIL cyclotron operation team for providing the primary beam. We also wish to express our appreciation for the vital contributions made by our late colleague and friend Jean-Marc Gautier to all the CHARISSA+DEMON experiments undertaken by our collaboration.

\section{Appendix: correlation formulas}

In the formalism of Ref. [8], the correlation function for neutrons of four-momenta $p_{i}$ emitted at a space-time relative distance $x=(\vec{r}, t)$ has two terms, originating respectively from Fermi statistics and the $s$-wave FSI, averaged over the distribution of distances:

$$
\begin{aligned}
& C_{n n}\left(p_{1}, p_{2}\right)=1+\left\langle b_{0}\right\rangle+\left\langle b_{i}\right\rangle \\
&\left\langle b_{0}\right\rangle=-\frac{1}{2}\langle\cos (q x)\rangle \\
&\left\langle b_{i}\right\rangle= \frac{1}{2}\left\{\left|f\left(k^{\star}\right)\right|^{2}\left\langle\left|\phi_{p_{1} p_{2}}(x)\right|^{2}\right\rangle\right. \\
&\left.+2 \Re\left[f\left(k^{\star}\right)\left\langle\phi_{p_{1} p_{2}}(x) \cos (q x / 2)\right\rangle\right]\right\}
\end{aligned}
$$

The metric is such that $p^{2}=|\vec{p}|^{2}-p_{0}^{2}$, the superscript * refers to the $2 n$ center of mass, $q=p_{1}-p_{2}$ is the relative four-momentum, $k^{\star}=\sqrt{q^{2}} / 2$ is the four-momentum of each neutron, and $f$ is their scattering amplitude:

$f\left(k^{\star}\right)=\left(-1 / a_{n n}+k^{\star 2} d_{0} / 2-i k^{\star}\right)^{-1}$

depending on the scattering length $a_{n n}$ and effective range $d_{0}$ (we use -18.5 and $2.8 \mathrm{fm}$, respectively [34). The $2 n$ wave function is factorized assuming $r^{\star} \gtrsim d_{0}$ as $f\left(k^{\star}\right) \phi_{p_{1} p_{2}}(x)$, with the exact form of $\phi_{p_{1} p_{2}}(x)$ given in Ref. [8]. However, note that the final expression of $C_{n n}$ (A8) does not depend on the form of $\phi_{p_{1} p_{2}}(x)$.

If we now assume a spherically symmetric source $W$ and neglect its momentum dependence:

$\left\langle b_{0}\right\rangle=-\frac{1}{2} \int W(x) \cos (q x) d^{4} x$ 


$$
\begin{array}{r}
\left\langle b_{i}\right\rangle=\int 2 \pi r_{T} d r_{T} d r_{L} d t W(x)\left\{\left|f\left(k^{\star}\right) \phi_{p_{1} p_{2}}(x)\right|^{2}\right. \\
\left.+2 \Re\left[f\left(k^{\star}\right) \phi_{p_{1} p_{2}}(x)\right] J_{0}\left(\frac{q_{T} r_{T}}{2}\right) \cos \left(q_{0} \frac{r_{L}-v t}{2 v}\right)\right\}
\end{array}
$$

with $L / T$ the directions parallel/perpendicular to the velocity $v$ of the pair. For a Gaussian source of the form $W(x)=\exp \left(-r^{2} / 4 r_{0}^{2}-t^{2} / 4 \tau_{0}^{2}\right)$ and small enough energies $\left(k^{\star} \ll m\right)$, after integration over $t^{\star}$ :

$$
\begin{aligned}
\left\langle b_{0}\right\rangle & =-\frac{1}{2} \exp \left(-4 k^{\star 2} r_{0}^{2}-q_{0}^{2} \tau_{0}^{2}\right) \\
\left\langle b_{i}\right\rangle & =\frac{1}{2 \sqrt{\pi} r_{0}^{2} \gamma \rho} \int r_{T} d r_{T} d r_{L}^{\star} \exp \left(-r_{T}^{2} / 4 r_{0}^{2}-r_{L}^{\star 2} / 4 \gamma^{2} \rho^{2}\right) \\
\times & \left\{\frac{|f|^{2}}{2 r^{\star 2}}+\Re\left[f \frac{\exp \left(i k^{\star} r^{\star}\right)}{r^{\star}}\right] J_{0}\left(\frac{q_{T} r_{T}}{2}\right) \cos \left(\frac{q_{0} r_{L}^{\star}}{2 \gamma v}\right)\right\} \\
& -(1 / 8 \sqrt{\pi})|f|^{2} d_{0} / \gamma \rho r_{0}^{2}
\end{aligned}
$$

with $\rho=\sqrt{r_{0}^{2}+v^{2} \tau_{0}^{2}}$. The last term in A8 is a firstorder correction of the integration of the expression used for $\phi_{p_{1} p_{2}}(x)$ in the region $r^{\star}<d_{0}$.

In the case of simultaneous emission and/or very small velocities $\left(\gamma \rho \approx r_{0}\right)$ the final expression becomes analytical, with only one free parameter $\left(r_{0}\right)$ :

$$
\begin{aligned}
C_{n n}\left(q_{n n}\right)=1 & -\frac{1}{2} \exp \left(-q_{n n}^{2} r_{0}^{2}\right)+\frac{|f|^{2}}{4 r_{0}^{2}}\left(1-\frac{d_{0}}{2 \sqrt{\pi} r_{0}}\right) \\
& +\frac{\Re f}{\sqrt{\pi} r_{0}} F_{1}\left(q_{n n} r_{0}\right)-\frac{\Im f}{2 r_{0}} F_{2}\left(q_{n n} r_{0}\right)
\end{aligned}
$$

with $F_{1}(z)=e^{-z^{2}} / z \int_{0}^{z} e^{x^{2}} d x$ and $F_{2}(z)=\left(1-e^{-z^{2}}\right) / z$. Otherwise one should use A7/A8, with two free parameters $\left(r_{0}, \tau_{0}\right)$. From the source parametrization used, one obtains $r_{n n}^{\mathrm{rms}}=\sqrt{6} r_{0}$ and $\tau=\sqrt{2} \tau_{0}$.

Note that this model cannot be applied for $r_{0} \lesssim$ $1 \mathrm{fm}\left(r_{n n}^{\mathrm{rms}} \lesssim 2.5 \mathrm{fm}\right)$, since then the result is completely determined by the short-distance behavior of $\phi_{p_{1} p_{2}}(x)$ in (A3), sensitive to the form of the $n$ - $n$ potential.

\section{References}

[1] A.S. Jensen et al , Rev. Mod. Phys. 76, 215 (2004).

[2] M.V. Zhukov et al , Phys. Rep. 231, 151 (1993).

[3] P. Mueller et al, Phys. Rev. Lett. 99, 252501 (2007).

[4] I.A. Egorova et al , Phys. Rev. Lett. 109, 202502 (2012).

[5] M.D. Jones et al, Phys. Rev. C 92, 051306(R) (2015).

6] K.M. Watson, Phys. Rev. C 88, 1163 (1952).

[7] A.B. Migdal, Sov. Phys. JETP 1, 2 (1955).

[8] R. Lednicky and V.L. Lyuboshits, Sov. J. Nucl. Phys. 35 770 (1982).

[9] K. Ieki et al, Phys. Rev. Lett. 70, 730 (1993).

[10] T. Nakamura et al, Phys. Rev. Lett. 96, 252502 (2006).

[11] F.M. Marqués et al , Phys. Lett. B 476, 219 (2000).

[12] F.M. Marqués et al, Phys. Rev. C 64, 061301 (2001).

[13] A. Revel et al , Phys. Rev. Lett. 120, 152504 (2018).

[14] D.Q. Fang et al , Phys. Rev. C 94, 044621 (2016).

[15] D.R. Tilley et al, Nuc. Phys. A 708, 3 (2002).

[16] F.M. Marqués et al, Nucl. Instr. Meth. A 450, 109 (2000).

[17] K. Markenroth et al , Nuc. Phys. A 679, 462 (2001).

[18] M. Nikolić, Kinematics and Multiparticle Systems, Gordon and Breach, (1968) p33.

[19] L.V. Grigorenko et al , Phys. Lett. B 677, 30 (2009).

[20] L.V. Grigorenko et al, Phys. Rev. C 80, 034602 (2009).
[21] L.V. Grigorenko, J.S. Vaagen, M.V. Zhukov, Phys. Rev. C 97, 034605 (2018).

[22] G. Normand, PhD Thesis, Université de Caen (2004), http://tel.archives-ouvertes.fr/tel-00008393

[23] M.S. Golovkov et al , Phys. Rev. C 72, 064612 (2005).

[24] J.K. Smith et al, Nuc. Phys. A 955, 27 (2016).

[25] A. Spyrou et al , Phys. Rev. Lett. 108, 102501 (2012); and F.M. Marqués et al , Phys. Rev. Lett. 109, 239201 (2012).

[26] B. Gabioud et al , Nuc. Phys. A 420, 496 (1984).

[27] M.A. Preston and R.K. Bhaduri, Structure of the Nucleus (Addison-Wesley, Reading, Massachusetts, 1975), p. 4550 .

[28] N. Colonna et al , Phys. Rev. Lett. 75, 4190 (1995).

[29] B.V. Danilin et al , Phys. Rev. C 69, 024609 (2004).

[30] Y. Yamaguchi et al, Phys. Rev. C 70, 054320 (2004).

[31] J.L. Lecouey et al, Phys. Lett. B 672, 6 (2009).

[32] A. Spyrou, Phys. Lett. B 683, 129 (2010).

[33] N.A. Orr et al , RIBF Experimental Proposal NP1106SAMURAI04.

[34] R.B. Wiringa, V.G.J. Stoks, R. Schiavilla, Phys. Rev. C 51, 38 (1995). 